\title{
How to retrieve a ruptured micro-catheter tip stuck in a tight lesion?
}

\author{
Hidemasa Shitan, Daisuke Hachinohe, Yoshifumi Kashima, Tsutomu Fujita \\ Cardiology and Catheterization Laboratories, Cardiovascular Medicine, \\ Sapporo Cardio Vascular Clinic, Sapporo Heart Center, Sapporo, Japan
}

\begin{abstract}
A 63-year-old male underwent percutaneous coronary intervention of the distal left circumflex artery due to effort angina. The target lesion showed critical stenosis with severe calcification and tortuosity. A 6-French guiding catheter was engaged to the left coronary artery via right radial artery. A SION blue wire (Asahi Intecc, Japan) could pass the lesion, however, a Caravel MC (Asahi Intecc, Japan) micro-catheter could not pass the lesion. When the Caravel MC was pulled out strongly, its tip was twisted off and left in the lesion.

Although there are some possible methods to retrieve it (e.g. use of a snaring catheter, twisted guide wire and so on), it was thought that these methods would not work well because of the very tight lesion.

It was thought that inserting a balloon with a tip diameter smaller than the ruptured tip might
\end{abstract}

enable retrieving both the balloon and the damaged tip.

First, a $1.0 \times 5 \mathrm{~mm}$ balloon was inserted but did not reach the ruptured tip. Consequently, a $1.25 \times 10 \mathrm{~mm}$ diameter balloon was inserted and pushed to the ruptured tip, then the balloon was attached to it. Then the connected tip and balloon catheter were carefully pulled out into the guiding catheter, and was successfully retrieved.

After crossing a Rotawire (Boston Scientific, USA), rotational atherectomy and balloon dilatation were performed, then a drug-eluting stent was implanted. The final angiography showed good results.

When a ruptured micro-catheter tip is stuck in a tight lesion, this balloon attached technique may be effective (Fig. 1, Suppl. Video 1).

Conflict of interest: None declared

Address for correspondence: Hidemasa Shitan, MD, Cardiology and Catheterization Laboratories, The Sapporo Heart Center, Sapporo Cardio Vascular Clinic, 8-1, Kita-49 Higashi-16, Higashiku, Sapporo 007-0849, Japan, tel: +81-11-784-7847, fax: +81-11-784-8400, e-mail: anti_samurai16@yahoo.co.jp

Received: 24.12.2018 Accepted: 17.01 .2020 

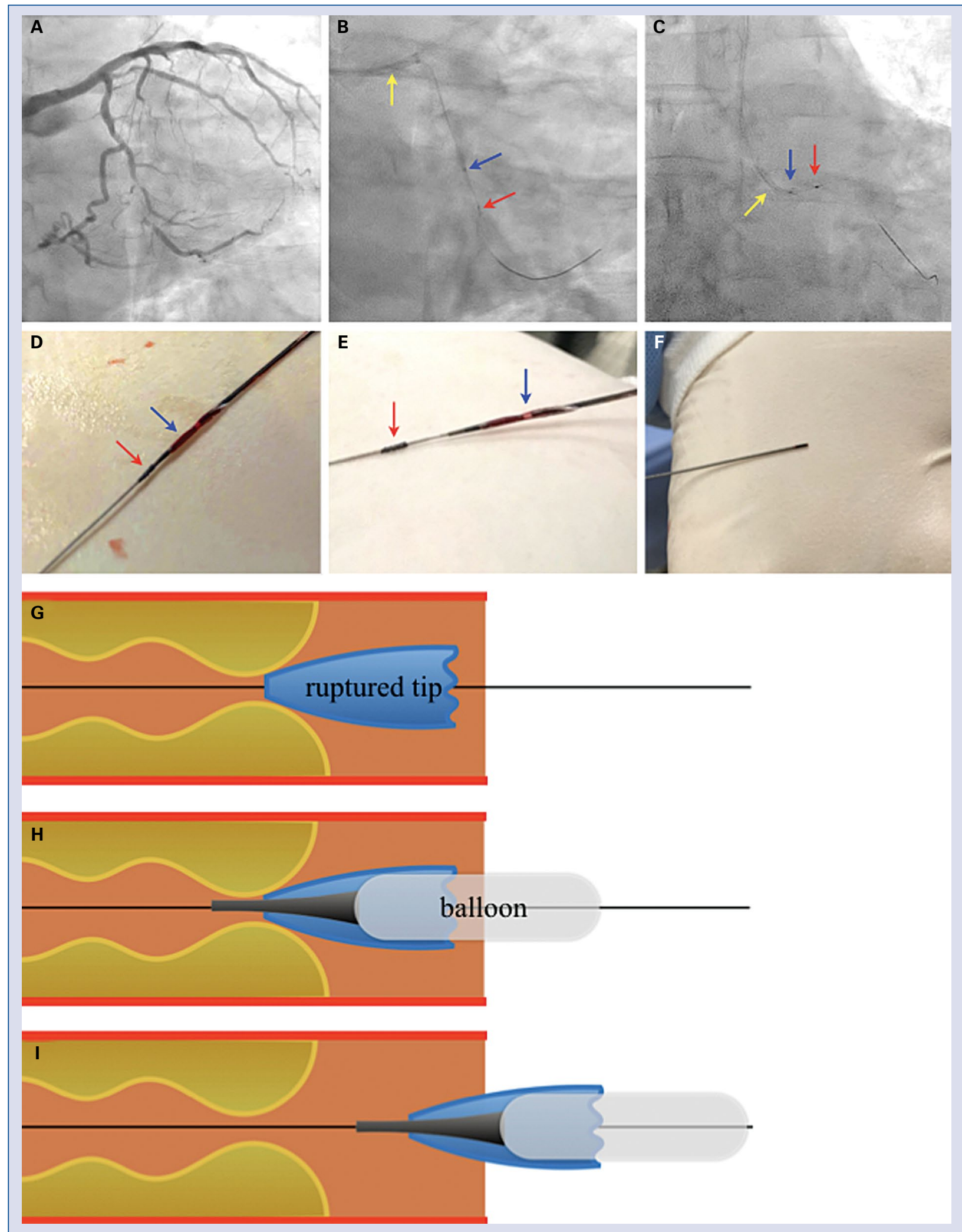

Figure 1. A. Initial angiography; B. Balloon is inserted and pushed to the ruptured tip; C. Retrieving the ruptured tip attached to the balloon into the guiding catheter; D. Ruptured tip attached to the balloon after retrieval from the guiding catheter; E. Ruptured micro-catheter tip is pulled out from the balloon tip; F. Ruptured micro-catheter; G. Illustration of micro-catheter left in a tight lesion; $\mathbf{H}$. Illustration of balloon which was inserted and attached to the ruptured tip; I. Illustration of ruptured tip retrieval using a balloon. Red arrow: a ruptured tip, blue arrow: a $1.25 \mathrm{~mm}$ balloon, yellow arrow: a guiding catheter. 